\title{
Multiwavelength observations of TANAMI sources
}

\author{
Felicia Krauß* \\ Dr. Karl Remeis Observatory \& ECAP, Sternwartstr. 7, 96049 Bamberg, Germany \\ Julius-Maximilian Universität Würzburg, Emil-Fischer-Str. 31, 97074 Würzburg, Germany \\ E-mail: Felicia.Krauss@sternwarte.uni-erlangen.de
}

\section{Cornelia Müller}

Dr. Karl Remeis Observatory \& ECAP, Sternwartstr. 7, 96049 Bamberg, Germany

Julius-Maximilian Universität Würzburg, Emil-Fischer-Str. 31, 97074 Würzburg, Germany

E-mail: Cornelia.Muelleresternwarte.uni-erlangen.de

\section{Matthias Kadler}

Julius-Maximilan Universität Würzburg, Emil-Fischer-Str. 31, 97074 Würzburg, Germany

\section{Jörn Wilms}

Dr. Karl Remeis Observatory \& ECAP, Sternwartstr. 7, 96049 Bamberg, Germany

\section{Moritz Böck}

Max-Planck-Institut für Radioastronomie,53010 Bonn, Germany

\section{Roopesh Ojha}

NASA, GSFC, Greenbelt, MD 20771 USA

\section{Eduardo Ros}

Universitat de València, 46100 Burjasot, Spain

Max-Planck-Institut für Radioastronomie, 53010 Bonn, Germany

\section{on behalf of the TANAMI collaboration}

The TANAMI VLBI program is monitoring a sample of 84 Active Galactic Nuclei of the Southern Sky at 8.4 and $22 \mathrm{GHz}$. The combination of VLBI and multiwavelength data allows us to study changes in the spectral energy distributions, as well as changes in the structure of the inner jets and to search correlations between both.

We present initial results of the multiwavelength analysis of a sub-sample of the TANAMI sources, combining our radio data with simultaneous X-ray and optical/UV observations from Swift and XMM-Newton, and gamma-ray data from Fermi, focusing on the broadband spectral energy distributions as well as variability in different wavebands.

11th European VLBI Network Symposium \& Users Meeting,

October 9-12, 2012

Bordeaux, France

${ }^{*}$ Speaker. 


\section{Introduction}

Active galactic nuclei (AGN) have been studied for over 100 years, however, many questions about their evolution and emission mechanisms remain to be answered. VLBI measurements can resolve emission regions on milliarcsecond scales which correspond to projected length scales of very few thousand Schwarzschild radii from the central black hole for close AGN. Our goal is to study the emission mechanisms of jets. We combine VLBI radio data with multiwavelength data from other instruments such as the Swift Gamma-ray Burst mission and the Fermi Gamma-ray Space Telescope. We use quasi-simultaneous data in order to establish a catalog for spectral energy distributions of a large sample of sources.

\section{The TANAMI Project}

Tracking Active Galactic Nuclei with Austral Milliarcsecond Interferometry (TANAMI) is a VLBI program monitoring a sample of sources south of -30 degrees declination since 2007, complementary to the MOJAVE project [7] on the Northern Hemisphere. The initial sample of 43 sources has been expanded to 84 since the launch of Fermi in 2008 [10]. These sources are monitored every $\sim 3$ months at 8.4 and $22 \mathrm{GHz}$ [12]. Figure 1 shows a map of the Southern Sky with the location of the TANAMI array. TANAMI is using the Australian Long Baseline Array (LBA+)

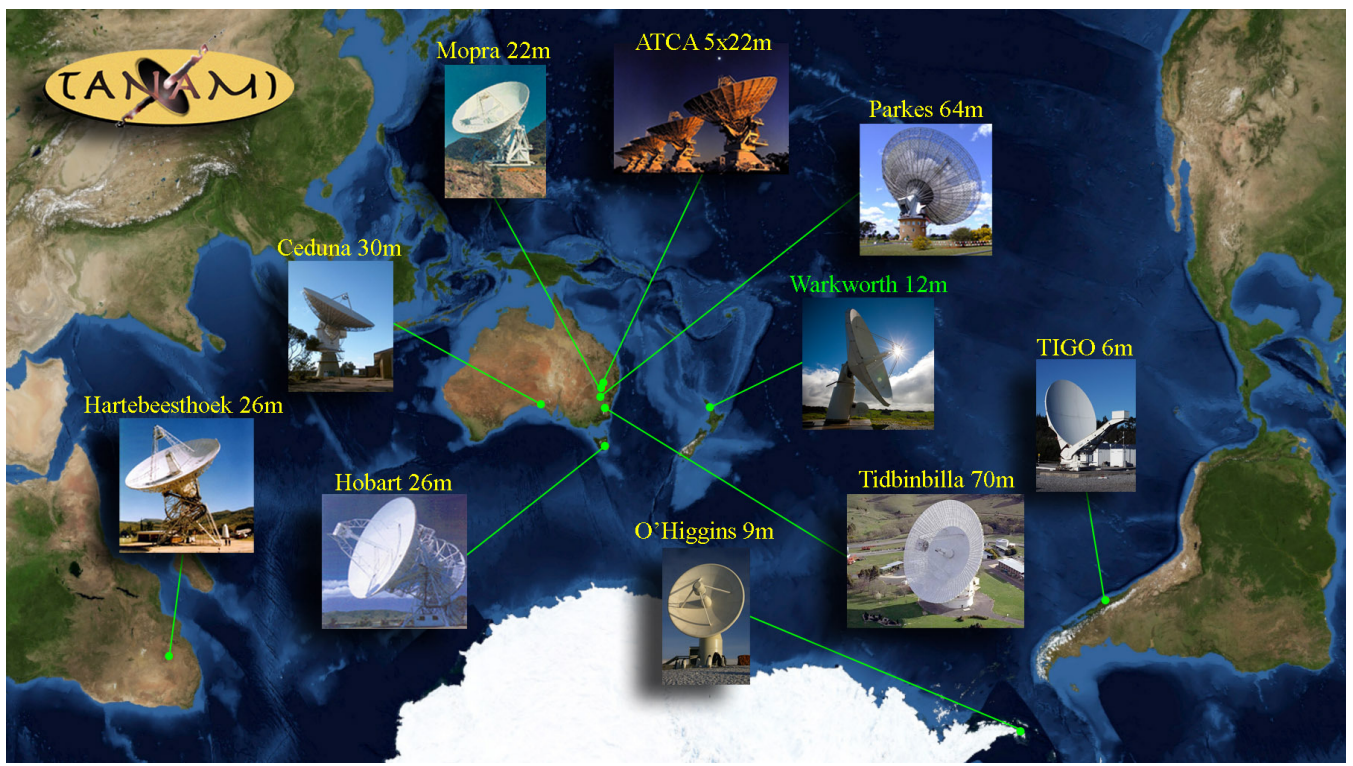

Figure 1: Map of the Southern Hemisphere showing the locations of the radio telescopes in the TANAMI Project.

with the antennas Parkes (64m), ATCA $(5 \times 22 \mathrm{~m})$, Mopra (22 m), Hobart $(26 \mathrm{~m})$, Ceduna $(30 \mathrm{~m})$ and the associated antennas Tidbinbilla (DSN $70 \mathrm{~m}$ or $34 \mathrm{~m}$ ), the IVS antennas TIGO (6 m, Chile) and O'Higgins (9 m, Antarctica), Hartebeesthoek (26 m, South Africa) and Warkworth (12 m, New Zealand). For a detailed discussion of the TANAMI data and analysis see also [8]. 


\section{Fermi/LAT light curve analysis and spectra}

AGN are very variable across the whole electromagnetic spectrum. Information about the source state is important to be able to create broadband spectra. The Fermi satellite is continuously monitoring the sky at $\gamma$-ray energies since its launch in 2008. Fermi observes in the $30 \mathrm{MeV}$ to $300 \mathrm{GeV}$ band [1]. This allows us to obtain light curves for all Fermi/LAT detected sources (C. Müller et al., in prep). 55 of the TANAMI sources have been detected by Fermi/LAT [4, 9]. As the Fermi analysis is still ongoing we used the public light curves which are available online. These light curves allow us to identify high and low states of a source.

The $\gamma$-ray bright object PKS 0537-441 shows strong variability in all wavebands. Figure 2 shows its $\gamma$-ray light curve including a bright flare in 2010. The colored areas show the time ranges

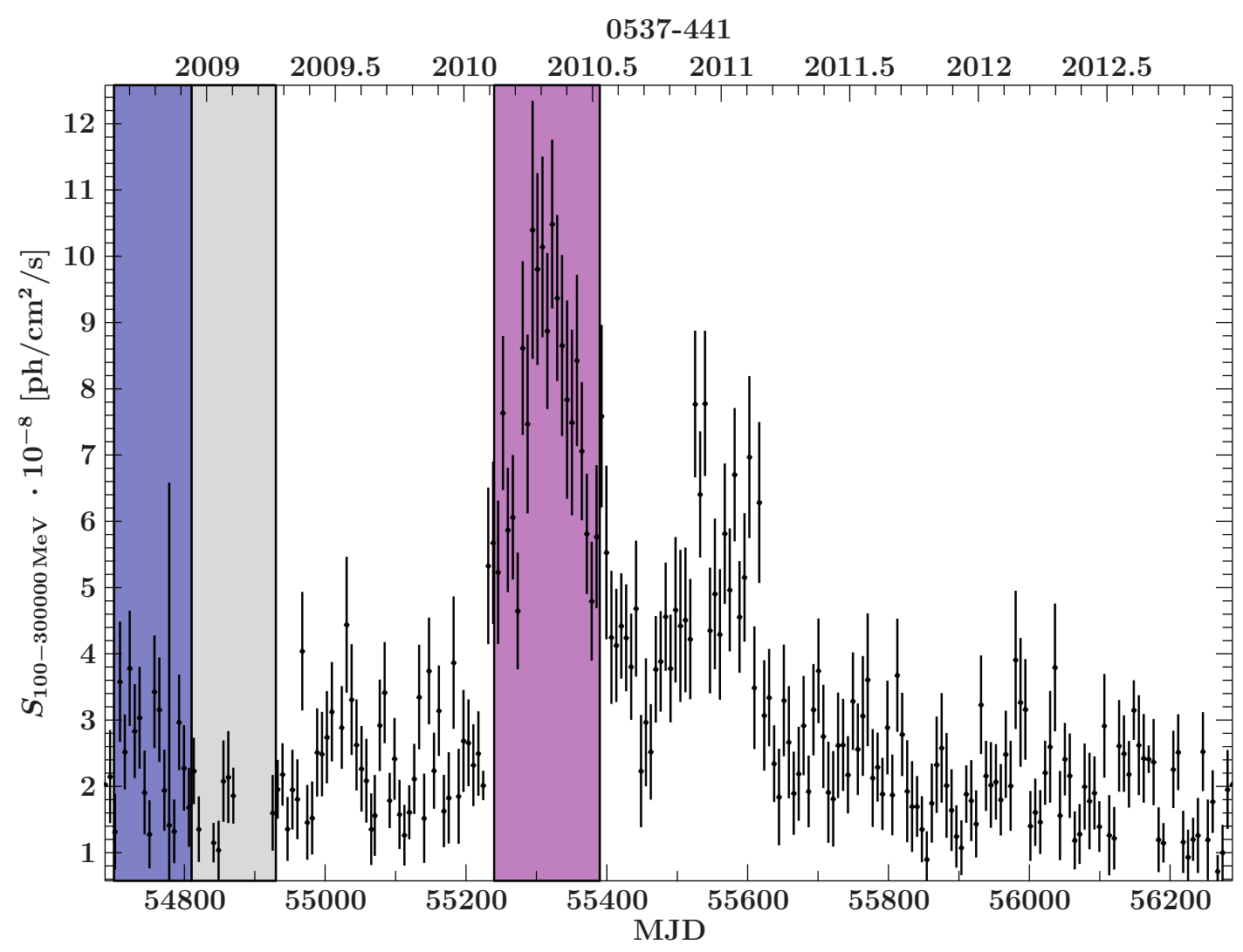

Figure 2: Identifying source states in the Fermi light curve of PKS 0537-441

used for creating quasi-simultaneous broadband spectral energy distributions (SEDs). The purple time range was identified as a high state, the blue area as an intermediate state and the gray area as a low state of the source.

The light curves show that quasi-simultaneous data are necessary for broadband spectra. Here we used spectral $\gamma$-ray information from the LAT 2-year point source catalog which contains flux information summed over the first 2 years of Fermi. The energy range of these spectra are in the $30 \mathrm{MeV}-300 \mathrm{GeV}$ Band. In the future we plan to create quasi-simultaneous spectra using Fermi data only in the time ranges given by the source state. 


\section{Multiwavelength Spectral Energy Distributions}

The broadband spectral energy distribution (SED) for PKS 0537-441 has been created from quasi-simultaneous TANAMI data (Kadler et al., in prep.) as well as Swift data (Optical/UV and $\mathrm{X}$-ray) $[5,6]$. The optical data have not been corrected for reddening and the Fermi/LAT data have not been corrected for the Extragalactic Background Light (EBL) absorption. In order not to falsify the data, the optical and X-ray data are not unfolded but modeled in detector space. The modeling of the SEDs with log parabolas is still ongoing and will be presented in a TANAMI SED catalog (Krauß et al., in prep.).

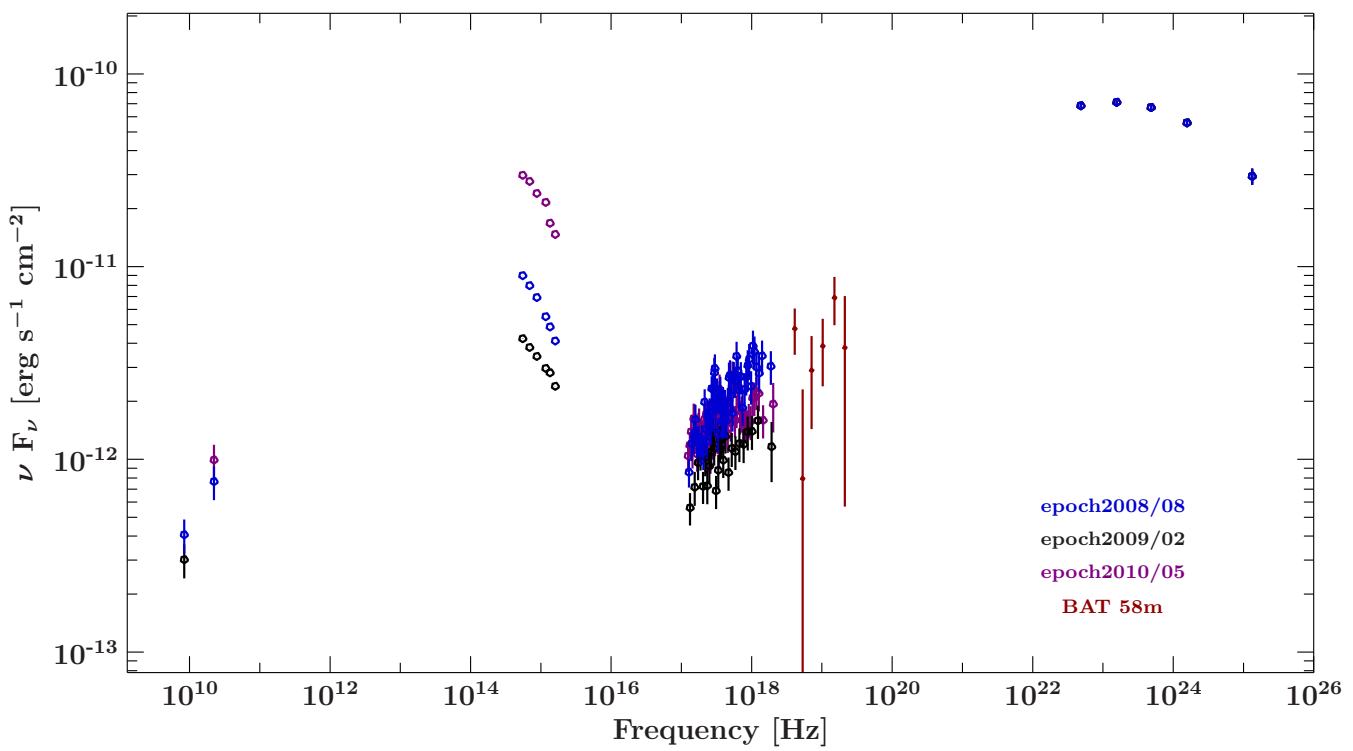

Figure 3: Spectral Energy Distribution of PKS 0537-441 including non-simultaneous 58-month Swift/BAT and 2FGL Fermi/LAT data

The colors of the TANAMI, Swift/UVOT and $S$ wift/XRT data in Figure 3 correspond to the colors used for identifying the source states in Figure 2. This figure also includes $S$ wift/BAT data from the 58 month catalog [2,3] and the Fermi data from the publicly available 2FGL catalog. [11]. The Swift/BAT and the Fermi data are not simultaneous as both data sets are summed over a large time range. Figure 3 also shows the variability that has been seen in the light curve. This is very apparent in the optical regime. The SED shows the double humped spectral form that is usually seen in blazars.

The low-energy peak is generally interpreted as synchrotron emission of electrons being accelerated in magnetic fields. The high-energy peak is usually associated with Synchrotron SelfCompton (SSC) emission. An alternative are hadronic or lepto-hadronic models. Spectral properties can be connected to the TANAMI VLBI information with two analysis methods. Highresolution images at two frequencies can be used to created spectral index maps of jets to pin down emission regions which are possible emission sites for $\gamma$-rays [8]. The continuous monitoring of the sources at radio and $\gamma$-ray frequencies allows us to study the radio- $\gamma$-ray connection even further. The ejection of jet features, revealed as traveling features at milliarcsecond resolution can 
be correlated with high states seen by Fermi and possible frequency-dependent time lags can be discerned [13].

\section{Results and future work}

We have given a short overview of the TANAMI project and presented initial results of a multiwavelength study of southern jets. This includes quasi-simultaneous broadband spectral energy distributions using TANAMI, $S$ wift/UVOT and $S$ wift/XRT data. Non-simultaneous $S$ wift/BAT and Fermi data have been added to the spectral energy distributions. Analysis of the publicly available Fermi light curves show the need for quasi-simultaneous coverage in all wavebands in order to study the different source states. Blazar variability and spectral energy distributions have to be studied in detail in the future and applied to a large source sample.

For a better understanding of the emission mechanisms of jets in active galactic nuclei more simultaneous multiwavelength campaigns are needed.

\section{Acknowledgments}

We thank the Deutsche Forschungsgemeinschaft for support under grant WI 1860/10-1. This research has made use of data and/or software provided by the High Energy Astrophysics Science Archive Research Center (HEASARC), which is a service of the Astrophysics Science Division at NASA/GSFC and the High Energy Astrophysics Division of the Smithsonian Astrophysical Observatory. This research has made use of the NASA/IPAC Extragalactic Database (NED) which is operated by the Jet Propulsion Laboratory, California Institute of Technology, under contract with the National Aeronautics and Space Administration. This research has made use of the SIMBAD database, operated at CDS, Strasbourg, France.

\section{References}

[1] Atwood W.B., Abdo A.A., Ackermann M., et al., 2009, Astrophys. J. 697, 1071

[2] Baumgartner W.H., 2011, The Swift-BAT 58 Month Survey, http://heasarc.gsfc . nasa.gov/docs/swift/results/bs58mon/

[3] Baumgartner W.H., Tueller J., Markwardt C., Skinner G., 2010, American Astron. Soci. 42, 675

[4] Böck M., Kadler M., Tosti G., et al., 2009, Proc. Fermi Symposium, eConf C091122

[5] Burrows D.N., Hill J.E., Nousek J.A., et al., 2005, Space Sci. Rev. 120, 165

[6] Gehrels N., Chincarini G., Giommi P., et al., 2004, Astrophys. J. 611, 1005

[7] Lister M.L., Homan D.C., 2005, AJ 130, 1389

[8] Müller, C., et al., "The TANAMI program: Southern-hemisphere AGN on (sub-)parsec scales", in Proceedings of 11th EVN Symposium, PoS (11th EVN Symposium) 020 
[9] Müller C., Böck M., Wilms J., et al., 2012, Proc. "Fermi \& Jansky", eConf C1111101

[10] Müller C., Kadler M., Ojha R., et al., 2011, Astron. Astrophys. 530, L11

[11] Nolan P.L., Abdo A.A., Ackermann M., et al., 2012, ApJS 199, 31

[12] Ojha R., Kadler M., Böck M., et al., 2010, Astron. Astrophys. 519, A45

[13] Sokolov A., Marscher A.P., McHardy I.M., 2004, Astrophys. J. 613, 725 